\title{
Analytic Calculation of Noise Power Robbing, NPR, and Polarization Isolation Degradation
}

\author{
Robert Peters* \\ Stellar Solutions, 250 Cambridge Ave., Palo Alto, CA 94306 \\ Peter Woolner \\ Noblis Inc. 3150 Fairview Park Drive South, Falls Church, VA 22042 \\ Ernest Ekelman ${ }^{*}$ \\ Perot Systems, 4500 Forbes Blvd, Suite 200, Lanham, MD 20706
}

\begin{abstract}
Three Geostationary Operational Environmental Satellite (GOES) R transponders (services) required analysis and measurements to develop an accurate link budget. These are a) Search and Rescue transponder which suffers from power robbing due to thermal uplink noise, b) the Data Collection Platform Report which suffers from degradation due to NPR (Noise Power Ratio), and c) GOES Rebroadcast transponder which uses a dual circular downlink $L$ band for which there was no depolarization data. The first two services required development of extended link budget to analytically calculate the impact of these degradations which are shown to have a significant impact on the link budget. The third service required measurements of atmospheric $L$ band $C P$ depolarization as there were no known previous measurements and results are reported here.
\end{abstract}

\section{Introduction}

The three transponders on the GOES R satellite that required additional effort to produce a viable link budget are discussed here. The Search and Rescue (SAR) transponder on the GOES R satellite will have a $100 \mathrm{kHz}$ bandwidth transponder and nominally ten $32 \mathrm{dBmi}, 400$ bps uplinks. Power robbing due to thermal noise is a well known phenomenon, but usually has a small arbitrary allocation in the link budget. However the SAR bandwidth is large compared to the occupied bandwidth and the very low, battery powered uplink results in a large amount of the HPA power being used to amplify noise. The C/No is shown to be reduced by over $7 \mathrm{~dB}$ due to power lost amplifying noise.

The Data Collection Platform Report (DCPR) has 250 FDMA/TDMA equivalent simultaneous carriers. Even with a relatively good noise power ratio (NPR) of $20 \mathrm{~dB}$, the 250 carrier DCPR transponder suffers almost $1 \mathrm{~dB}$ of loss due to NPR. The trade-off of NPR vs. the RF power to maintain a fixed link margin is explored since HPA NPR can be improved at the cost of loss of RF power due to higher output power back-off. A NPR sweat spot of 17 to $22 \mathrm{~dB}$ is found for this specific link.

The GOES Rebroadcast (GRB) has an X band dual polarization uplink and a dual polarization downlink at L band. The treatment of depolarization effects is very different for the two cases. In addition, there were no known measurements of atmospheric depolarization effects at $\mathrm{L}$ band. To develop requirements for this link, depolarization measurements were made and a link budget developed which incorporated the differences in environmental depolarization in the uplink and downlink.

* Goddard Space Flight Center, Greenbelt, Maryland 20771 


\section{Noise Power Robbing-SAR Transponder}

Power robbing can significantly impact the link budget for very low power uplinks and a relatively wide low occupied bandwidth. The SAR transponder of the GOES R satellite will have is such a system with a few $32 \mathrm{dBm}$, $400 \mathrm{kbps}$ uplinks from small battery powered beacons transmitters and a $100 \mathrm{kHz}$ bandwidth. The relatively wide bandwidth is required because the beacons are activated as needed in emergencies and it is necessary to avoid collisions of uplinks. The uplink power is further reduced by a $4.1 \mathrm{~dB}$ polarization loss.

To calculate the power lost to noise, we use the usual definitions of noise/Hz is No, the value of the noise over the HPA bandwidth is $\mathrm{N}$, the total carrier power is $\mathrm{Co}$, the carrier power after power lost to noise is $\mathrm{C}$.

Let $\mathrm{x}=\mathrm{C} / \mathrm{N} ; \mathrm{N}=\mathrm{C} / \mathrm{x}$

$\mathrm{Co}=\mathrm{C}+\mathrm{N}=\mathrm{C}(1+1 / \mathrm{x})$

$\mathrm{C} / \mathrm{Co}=1 /(1+1 / \mathrm{x})=1 /(1+\mathrm{N} / \mathrm{C})$

For the GOES link, the energy lost to amplifying noise on the Search and Rescue (SAR) link is substantial. The UHF uplinks (406.05 MHz) low power uplinks have a minimum polarization loss of $4.1 \mathrm{~dB}$. Using estimated characteristics and assuming 10 simultaneous uplinks, amplifying the noise decreases the power available for the signals by $7.6 \mathrm{~dB}$.

\begin{tabular}{|l|r|}
\hline Total EIRP $(\mathrm{dBm})$ & 51.08 \\
\hline Carrier power robbing due to noise $(\mathrm{dB})$ & -7.59 \\
\hline Power sharing with other carriers & -10.00 \\
\hline NET EOC EIRP/carrier (dBm) & 33.49 \\
\hline
\end{tabular}

Figure 1 Power lost to noise in the SAR transponder

\section{Noise Power Ratio - DCPR Transponder}

The GOES-R DCPR transponder is designed for 250 (equivalent) simultaneous shared circuits in an FDMA/TDMA configuration. A significant source of degradation of this link is due to the Noise Power Ratio (NPR). A satellite transponder, or other amplifier, is loaded with a white noise input over its full operating bandwidth, except for small test channel or notch, the input noise is filtered out. The total input power must be large enough to drive the output amplifier to its rated operating power level. The output signal is examined and the noise power measured within the notch and, generally, at several frequencies elsewhere in the transponder passband. Each noise power measurement must be made using the same bandwidth, which must therefore be narrow enough to provide an accurate measurement of the depth of the notch. The several high power measurements are averaged and the result is compared to the power level measured within the notch, thus producing the ratio of these two noise powers, the NPR, which is expressed in $\mathrm{dB}$.

NPR test may be thought of as the limiting case when an infinite number of infinitely narrow signals (noise) are passed through the limited bandwidth of the transponder. Some fraction of that noise is translated into the notch due to intermodulation products from the imperfect linearity of the amplifiers through which the noise is passed. This is equivalent to the sum of all intermodulation products, between all the possible combinations of signals that produce a product, third, fifth, seventh, etc. that will fall into the narrow band of that slot.

When the actual number of signals to be carried by a transponder is large, the NPR test will provide a more realistic measurement of the non-linearity effects due than can be produced by a simple carrier to third order intermodulation product (C/3IM) since the effects of all possible combinations of input signals and all their intermodulation products are captured in this one set of measurements under a single set of test conditions. 
An example of an NPR input and output spectral display is shown in Figure 2, below, created using MatLab. In this example the input spectrum and the output spectrum is shown. The NPR measurement result is shown in the central notch at approximately $12 \mathrm{~dB}$. Note that the notch depth in the input signal is approximately $16 \mathrm{~dB}$ below the notch depth in the output signal, which shows that the noise level in the notch is almost completely due to the channel nonlinearity with only about $0.1 \mathrm{~dB}$ due to the input noise in the notch bandwidth.

For an actual NPR test, the bandwidth of the notch would usually be a much smaller percentage of the total occupied bandwidth than is shown here, where the notch width was exaggerated to more clearly show the input and output levels. Unless the notch bandwidth is a negligibly small fraction of the total bandwidth, e.g. one percent or less, the NPR level in the notch will show a slope down and away from the edges of the high-noise bands, as is shown at the high and low frequency ends of this display.

The raw NPR measurement is not a parameter that can be directly used in the link analysis, but can be converted into a ratio that can be included. i.e. the ratio of carrier power, $\mathrm{C}$, to the NPR per hertz, $\mathrm{NPR}_{0}$. This $\mathrm{C} / \mathrm{NPR}_{0}$ is then easily combined with the usual link parameter, $\mathrm{C} / \mathrm{N}_{0}$ for the up and down parts of the total link budget.

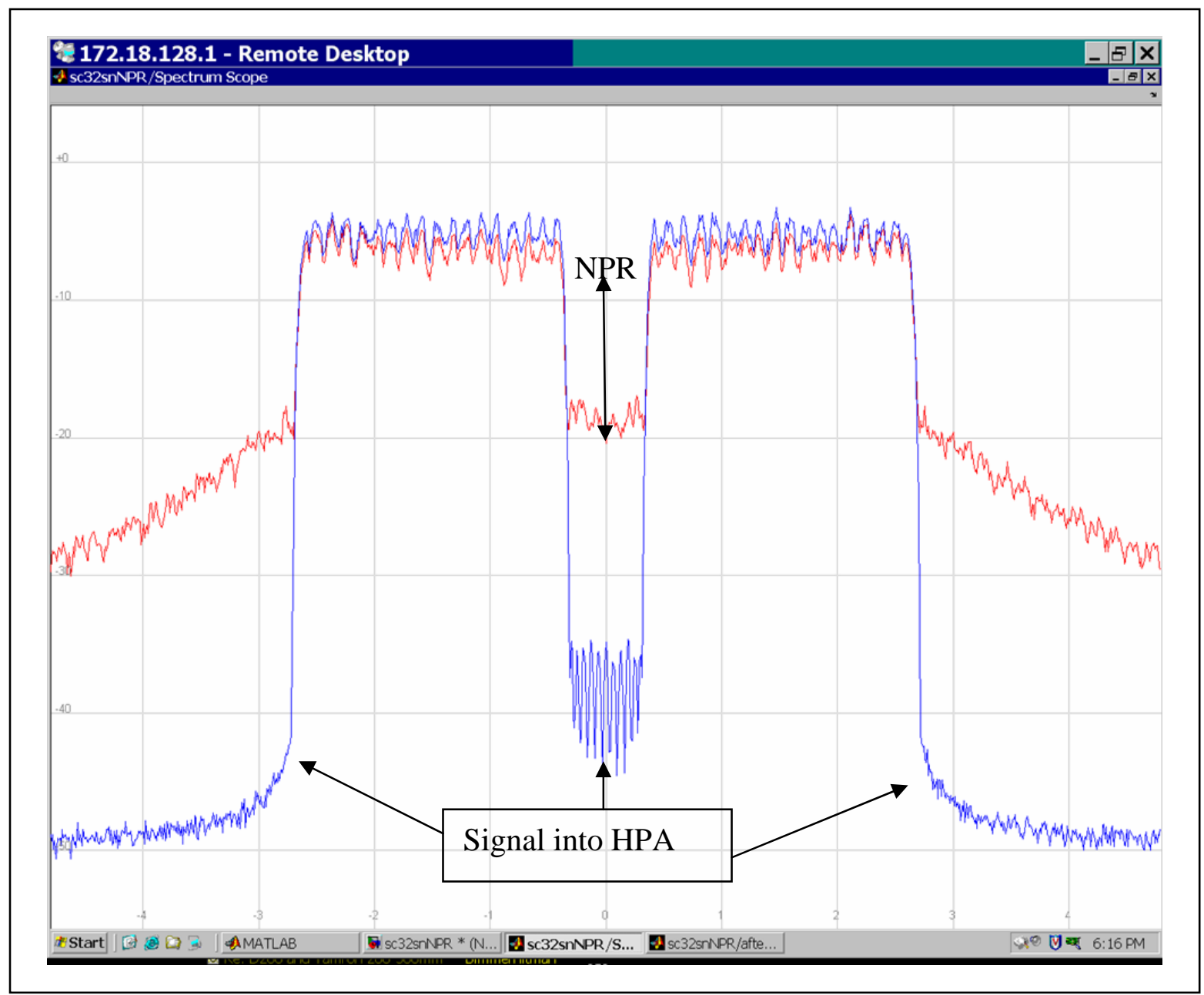

Figure 2 Example Input (blue, lower trace) and Output Spectral Display (red, upper trace).

The NPR is valid for any measuring bandwidth, provided it is the same in the notch and for the high power measurements and is able to adequately reject the adjacent high power when it is tuned within the notch. Therefore that ratio also applies to a $1 \mathrm{~Hz}$ bandwidth. However, if there are $\mathrm{N}$ signals to be transmitted in a bandwidth $\mathrm{B} \mathrm{Hz}$, all at the same or average level, then the power in each carrier, C, is greater than the power in $1 \mathrm{~Hz}$ of high level 
noise by a factor of $\mathrm{B} / \mathrm{N}$, i.e. the power in each carrier is equal to the sum of the noise power over that fraction of the total bandwidth allocated to that carrier. Therefore:

$$
\mathrm{C} / \mathrm{NPR}_{0}=\mathrm{NPR}+10 * \log (\mathrm{B} / \mathrm{N}) \mathrm{dB} / \mathrm{Hz}
$$

For carriers that are not at the average level, the $\mathrm{C} / \mathrm{NPR}_{0}$ must be adjusted by the same amount that each signal level differs from the average; i.e. if a given signal is $2 \mathrm{~dB}$ less than average, the NPR is $2 \mathrm{~dB}$ less than was calculated for the average signal level. The NPR can therefore be incorporated into the link calculations for any signal at any level.

The NPR parameter is particularly appropriate for the DCPR transponder on the GOES satellites, and incorporating the NPR results into the link budget is considered necessary to accurately model the effects of the transponder on the end-to-end performance of individual signals. The details of how the individual transmitters are allocated by NOAA to specific channels within the $400 \mathrm{kHz}$ DCPR transponder bandwidth are not relevant to this paper. However, NOAA has defined the maximum loading is equivalent to 250 simultaneous signals at an average uplink transmit EIRP level of $41 \mathrm{dBmi}$ and all channels are defined with a nominal $750 \mathrm{~Hz}$ center-to-center spacing and almost all active channels are grouped together in the lower $300 \mathrm{kHz}$ of the band. A typical transmission is expected to take about 10 seconds, but as there will be 30 to 50 thousand Data Collection Platforms, transmitting on average 24 times per day, the defined maximum loading will be closely approached during the most popular use periods.

Almost all of these signals will be 8-PSK modulated at 300 bps with rate 2/3 Trellis coding and therefore will occupy a null-to-null bandwidth of $300 \mathrm{~Hz}$. In actual operation, some signals passing through the transponder will be 100 bps BPSK and some will be 1200 bps 8-PSK, but they are only a small percentage of the total. These differences are only significant when it is necessary to calculate the performance of one of these unusual signals; they were included in the calculation of the above number of signals and average power.

The DCPR transponders on the GOES-R series satellites will have a passband of $400 \mathrm{kHz}$ and a specified NPR or 20 $\mathrm{dB}$. The minimum uplink EIRP required to produce an acceptable $\mathrm{E}_{\mathrm{B}} / \mathrm{N}_{0}$ is $37 \mathrm{dBmi}$, which applies to a $300 \mathrm{bps}$ signal and the maximum is $47 \mathrm{dBmi}$ which is only permitted for a 1200 bps signal.

When we insert the appropriate values in the previous equation, we get:

$$
\begin{aligned}
\mathrm{C} / \mathrm{NPR}_{0}= & 20+10 * \log (400000 / 250) \mathrm{dB} / \mathrm{Hz} \\
& =52.04 \mathrm{~dB} / \mathrm{Hz}
\end{aligned}
$$

As this value applies to an average uplink, the $\mathrm{C} / \mathrm{NPR}_{0}$ for a minimum uplink will be $4 \mathrm{~dB}$ less, $48.04 \mathrm{~dB} / \mathrm{Hz}$ and for a maximum uplink it will be $6 \mathrm{~dB}$ greater, $58.04 \mathrm{~dB} / \mathrm{Hz}$.

The parameter $\mathrm{C} / \mathrm{NPR}_{0}$ can be added to the $\mathrm{C} / \mathrm{N}_{0}$ values typically derived for the up and down parts of a satellite link. Indeed, $\mathrm{C} / \mathrm{NPR}_{0}$ can be added to either the up or down $\mathrm{C} / \mathrm{N}_{0}$ value, or all three may be added together in one calculation, because the equation for adding non-coherent powers is associative.

As an example, if the uplink EIRP is $37 \mathrm{dBmi}$, a typical DCPR link calculation could produce an uplink plus downlink $\mathrm{C} / \mathrm{N}_{0}$ of $40.92 \mathrm{~dB} / \mathrm{Hz}$. When the $\mathrm{C} / \mathrm{NPR}_{0}$ of $48.04 \mathrm{~dB} / \mathrm{Hz}$ is added, the overall downlink $\mathrm{C} /\left(\mathrm{N}_{0}+\mathrm{NPR}_{0}\right)$ is $40.15 \mathrm{~dB} / \mathrm{Hz}$.

This is illustrated Figure 3. For this transponder, the loss of power due to noise power robbing is negligible, about $0.16 \mathrm{~dB}$.

\begin{tabular}{||l|r|}
\hline Net antenna EOC qain (dBi) & 11.70 \\
\hline EOC EIRP all carriers (dBm) & 48.72 \\
\hline Power sharing 37 dBm to 249 other carriers (dB) & -27.98 \\
\hline Carrier power robbing due to noise (dB) & -0.16 \\
\hline Net EOC EIRP for 37 dBm Carrier $(\mathbf{d B m})$ & 20.59 \\
\hline NPR $(\mathrm{dB})$ & 20.00 \\
\hline C/lo $(\mathrm{dB} / \mathrm{Hz})$ & 48.04 \\
\hline
\end{tabular}




\begin{tabular}{|l|r|}
\hline Downlink C/No $(\mathrm{dB} / \mathrm{Hz})$ excluding NPR & 40.93 \\
\hline Downlink $\mathrm{C} / \mathrm{No}_{\mathrm{o}}(\mathrm{dB} / \mathrm{Hz})$ including NPR & 40.16 \\
\hline
\end{tabular}

Figure 3 Reduction in C/N due to NPR degradation



Figure 4 Impact of NPR on C/No at constant power and required downlink power to maintain constant C/No.

The overall results of varying the NPR on the end-to-end link design are shown in two different ways in Figure 4. First, if the NPR is reduced from $40 \mathrm{~dB}$, the curve labeled $\mathrm{C} / \mathrm{N}_{0}$, (where $\mathrm{N}_{0}$ includes the impact of $\mathrm{NPR}_{0}$ ) shows there is about $0.1 \mathrm{~dB}$ degradation when the NPR is near $30 \mathrm{~dB}$ and about $1 \mathrm{~dB}$ degradation when the NPR is near 20 $\mathrm{dB}$. The degradation starts to increase more rapidly as the NPR is reduced below $20 \mathrm{~dB}$. Alternately the impact of NPR can be shown as the RF power necessary to maintain a constant $E_{B} / N_{0}$ of $13 \mathrm{~dB}$ as the NPR decreases, as also shown in Figure 4. (This applies to a specific set of assumed link parameters where the required $E_{B} / N_{0}$ is $11 \mathrm{~dB}$.) The optimum trade-off depends upon the characteristics of the HPA, but should be between 17 and $22 \mathrm{~dB}$ for this link where the required $\mathrm{Eb} / \mathrm{No}$ is $13 \mathrm{~dB}$.

\section{Polarization Degradations - GOES Rebroadcast (GRB) transponder}

The GRB X band uplink will be from a 16 meter antenna at the NOAA Command and Data Acquisition Station at Wallops Is. The L band downlink is to earth stations over the hemisphere. The atmospheric depolarization and the type of receive stations resulted in the selection of linear polarization for the uplink and circular polarization for the downlink. The calculation of depolarization effects is substantially different for the two cases. Atmospheric circular depolarization has not been measured at $\mathrm{L}$ band and measurements had to be made to obtain values to use in the link budget. The initial results are presented in this section. 
For dual linear polarization, alignment of the ground antenna to match the satellite antenna is accomplished by rotating the ground antenna polarization until the cross polarized signal is minimized. In clear weather this will provide polarization isolation equal to the satellite antenna cross polarization discrimination or the ground antenna cross polarization discrimination which ever is worse. For the link budget calculation, the worst case polarization discrimination of the ground or satellite antenna and the atmospheric depolarization should be used. Since NOAA has no control over the receive stations it can not assume that the receive antenna will be rotated to minimize cross polarization. Since rotating the feed minimizes the cross polarization component due to the antennas, the worst uplink contributor is expected to be due to the atmosphere and be better than $25 \mathrm{~dB}$ so this value is used to calculate the degradation to the uplink carrier-to-noise.

Dual circular polarization was selected for the GRB L band downlink for two reasons. First, the ability to rotate the antenna polarization is not commonly provided and if it were, NOAA cannot assume the ground receive stations would take advantage of it. Therefore the antenna cross polarization cannot be minimized by aligning the transmit and receive polarizations. Second, circular polarization suffers less atmospheric depolarization than linear polarization at L- band (the opposite is true at $\mathrm{X}$ band). At L band, Faraday rotation (the effects through the ionosphere which are sun dependent) can rotate the linearly polarized signal as much as 16 to 20 degrees making it impractical for dual polarization frequency reuse. For circular polarization, the worst case polarization isolation occurs when the cross polarization discrimination from all the contributing sources (transmit antenna, receive antenna, and atmospheric depolarization) combine destructively. The equivalent worst case isolation is found by adding the voltages from the three downlink contributors to cross polarization; the transmit cross polarization, atmospheric contributions, and receive antenna cross polarization. Assuming that the energy lost to the interfering polarization is small so that the carrier power is fairly constant:

$$
\text { (Isol)down }=-20 * \mathrm{LOG}\left[10^{\wedge}\left(-\mathrm{I}_{\mathrm{Tx}} / 20\right)+10^{\wedge}\left(-\mathrm{I}_{\mathrm{Rx}} / 20\right)+10^{\wedge}(-(\mathrm{Patmos} / 20)]\right.
$$

Where $\mathrm{I}_{\mathrm{Tx}}$ is the spacecraft transmit isolation, $\mathrm{I}_{\mathrm{Rx}}$ the ground receive isolation, Patmso the atmospheric depolarization, all in $\mathrm{dB}$.

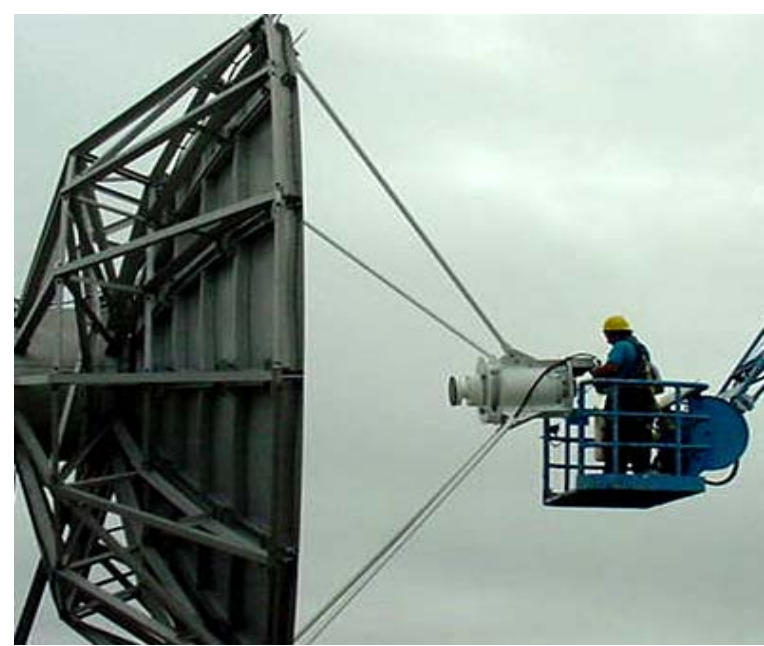

Figure 5 7.2m Antenna with new rotating $C P$ feed with $0.2 \mathrm{~dB}$ axial ratio
Then the effect on the carrier to noise can be found

$\mathrm{C} / \mathrm{No}=\left[1 /(\mathrm{C} / \mathrm{No})+1 / \mathrm{C} /\left(\mathrm{I}_{\mathrm{Tx}}\right)+1 /\left(\mathrm{C} / \mathrm{I}_{\mathrm{Rx}}\right)+1 /(\mathrm{Isol})_{\mathrm{down}} / \mathrm{BR}\right]^{-1}$

where BR is the data bit rate. All values must be linear, not logarithmic.

Unfortunately, no current geosynchronous satellites are using dual polarization at L-band, so the weather effects are generally unknown below $4 \mathrm{GHz}$. Another problem involves Faraday rotation through the atmosphere. Faraday rotation, the effects of the Ionosphere, is much worse at L-band than at $4 \mathrm{GHz}$ and this causes linear signals to rotate in orientation. Using dual circular polarization at L-band for the GRB downlink will solve that problem. The circularly polarized ellipse vectors for the orthogonally polarized right-hand circular polarization (RCP) and left-hand circular polarization (LCP) may rotate with respect to each other but if the axial ratios are sufficiently good the polarization discrimination degradation will be acceptable.

To verify that the degradation is acceptable using dual circularly polarized signals at L-band, a two part measurement program was initiated. In the Lab receiver testing demonstrated that when using QPSK modulation and LDPC coding, an Eb/No of $6.5 \mathrm{~dB}$ (which includes implementation loss) is required. This link will tolerate 12 $\mathrm{dB}$ of polarization isolation and maintain the required $2.5 \mathrm{~dB}$ margin. The second measurement program involved monitoring a satellite transmitted downlink RCP signal in both the RHC and LHC polarizations of a single CP signal. Comparison of received RCP/LCP signal power levels, relative to existing weather conditions provided 
information on the polarization isolation that could be achieved. A new dual polarized feed with excellent axial ratio and the capability to rotate was designed, fabricated, tested, and installed in an available 7.2m antenna at Wallops for carrying out the monitoring phase (Figure 5).

The Search and Rescue (SAR) RHCP signal transmitting at $1544.5 \mathrm{KHz}$ from the GOES 11 satellite located at $135^{\circ}$ West longitude was used as a stable source. This signal was chosen for its excellent axial ratio of $0.41 \mathrm{~dB}(32.5 \mathrm{~dB}$ cross polar discrimination) and its $15^{\circ}$ look angle with a long slant path through the atmosphere providing opportunities to measure numerous bad weather events. The system cross polarization isolation in good weather was measured by rotating the feed though more than $180^{\circ}$ and recording the variation in cross polarization level received in the LHCP port. By using the maximum ( $40 \mathrm{~dB})$ and the minimum $(29 \mathrm{~dB})$ levels during the rotation as the polarization ellipses combine in and out of phase it is possible to calculate the axial ratio/cross polar discrimination of the satellite antenna $(0.41 \mathrm{~dB} / 32.5 \mathrm{~dB})$ and the $7.2 \mathrm{~m}$ ground antenna $(0.22 \mathrm{~dB} / 38 \mathrm{~dB})$. The feed was rotated for maximum cross polarization isolation during monitoring to provide the best sensitivity for measuring the effects of the atmosphere. A computer based automated measurement was developed to record the data. Using data from Doppler weather radar available from National Mosaic and Quantitative Precipitation Initiative (NMQ), the magnitude of atmospheric rain in the link path was determined. Many rain events were recorded and sample plots of the results are shown in Figure 6 which shows that the isolation varies some even during good weather at the 37 to $43 \mathrm{~dB}$ down level and follow a daily cycle as shown by the orange curve which is the average level for the month of March. During one of the most sever rain events recorded to date the rain rate exceeded the 99.99\% (53 minutes per

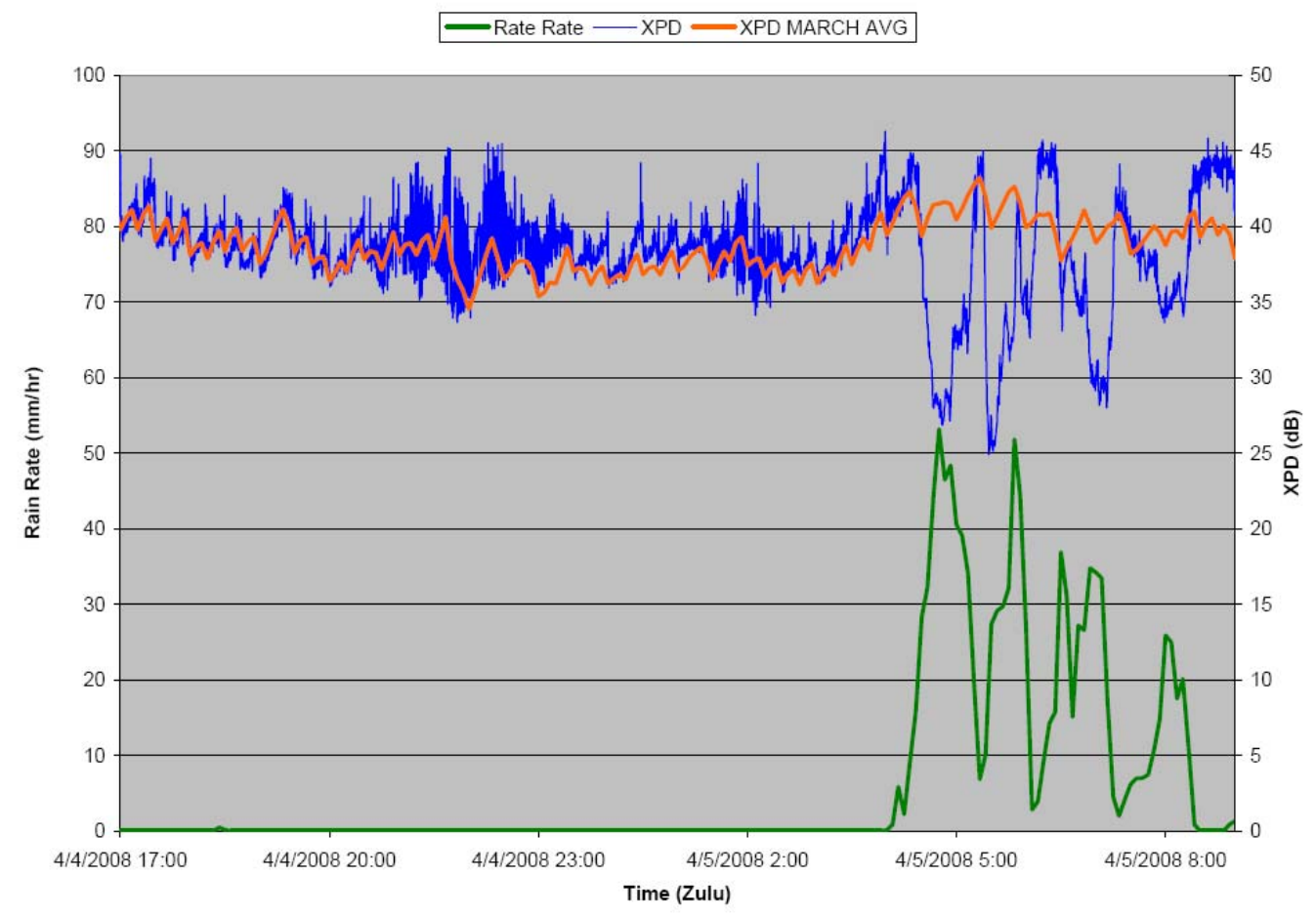

Figure 6 Measured cross polarization isolation before and during a very strong rain event and showing rain rate and a monthov average.

year) worst case annual rate of $45.7 \mathrm{~mm} /$ hour for Wallops, VA. The cross polarization isolation degraded to $25 \mathrm{~dB}$ and improved to $45 \mathrm{~dB}$ as a direct result of the atmospheric rain. To be conservative, the worst case expected isolation was picked to be $22 \mathrm{~dB}$ for the L-band downlink time correlated with the measured system isolation. Monitoring started in October 2007 and for GOES-R. The satellite antenna was specified at $0.55 \mathrm{~dB}$ axial ratio (30 $\mathrm{dB}$ XPD) and the numerous ground antenna were specified at $0.78 \mathrm{~dB}$ axial ratio (27 dB XPD). Combining these values using the equations above the worst case expected polarization isolation will be $16.2 \mathrm{~dB}$, sufficient to maintain the required margin. 


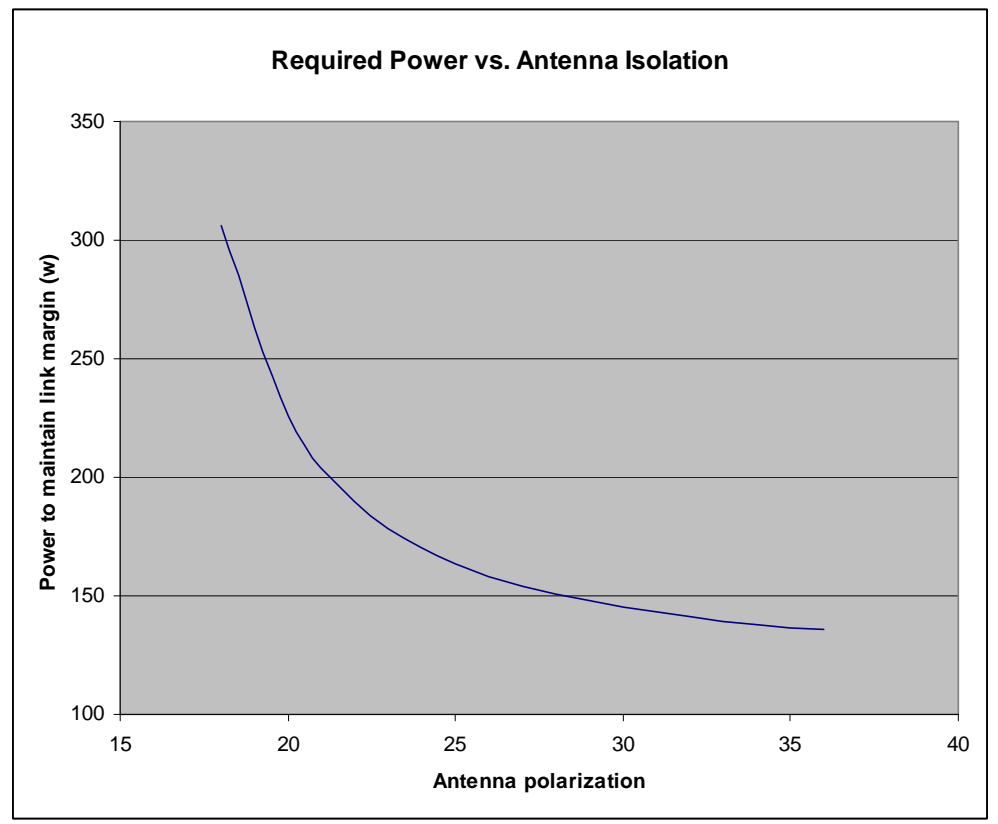

The required $\mathrm{Eb} / \mathrm{No}$ with coding is about 4.5 $\mathrm{dB}$ and an additional link margin of $2.5 \mathrm{~dB}$ is specified. The spacecraft RF power required under clear sky conditions and assuming a 2.7 BOL margin, and assuming various transponder values is about $115 \mathrm{~W}$. If there is an atmospheric depolarization of $22 \mathrm{~dB}$, the required RF power increases to $145 \mathrm{~W}$ using the parameters previously given. More generally, the required downlink RF power was plotted in Figure 7 as a function of the spacecraft antenna isolation. A sample link budget showing the up, down, and combined $\mathrm{C} / \mathrm{No}$ (which includes interference) is shown in Figure 8 which shows the downlink link degradation of about $1.3 \mathrm{~dB}$ due to polarization degradation.

Figure 7 Power required to maintain link margin vs. spacecraft polarization isolation

\begin{tabular}{||l|r|}
\hline Downlink C/No (dB/Hz) & 83.12 \\
\hline I downlink polarization interference $(\mathrm{dB})$ & 16.15 \\
\hline Downlink $\mathrm{C} /(\mathrm{No}+\mathrm{lo})(\mathrm{dB} / \mathrm{Hz})$ & 81.91 \\
\hline Uplink $\mathrm{C} / \mathrm{N}(\mathrm{dB} / \mathrm{Hz})$ & 89.42 \\
\hline Total Link $\mathrm{C} / \mathrm{No}(\mathrm{dB} / \mathrm{Hz})$ & 81.20 \\
\hline
\end{tabular}

Figure 8 GRB C/(No+I)

\section{Conclusions}

Link budgets usually have some values which are estimated, not calculated. This is usually adequate for most purposes but three transponders on the GOES satellite required additional work to develop a viable link budget. We have derived exact equations to calculate the impairments due to NPR and to noise power robbing and found that the calculated values result in significant corrections to the GOES R satellite link budget. The use of dual polarized L band downlinks required new measurements of depolarization in that frequency band and very different treatment of the uplink and downlink impairments. 